\title{
Compter pour compter
}

Les manifestations pratiques de savoirs criminologiques dans les instances locales de sécurité

Counting for the sake of counting. The pratical manifestations of a criminological knowledge in local organisations dedicated to security

\section{Élodie Lemaire et Laurence Proteau}

\section{(2) OpenEdition \\ Journals}

\section{Édition électronique}

URL : http://journals.openedition.org/conflits/18882

DOI : $10.4000 /$ conflits. 18882

ISSN : $1777-5345$

Éditeur :

CCLS - Centre d'études sur les conflits lilberté et sécurité, L'Harmattan

Édition imprimée

Date de publication : 31 décembre 2014

Pagination : 43-64

ISBN : 978-2-343-05760-6

ISSN : 1157-996X

\section{Référence électronique}

Élodie Lemaire et Laurence Proteau, « Compter pour compter », Cultures \& Conflits [En ligne], 94-95-96 | été-automne-hiver 2014, mis en ligne le 20 février 2016, consulté le 30 mars 2021. URL : http:// journals.openedition.org/conflits/18882 ; DOI : https://doi.org/10.4000/conflits.18882 


\section{Compter pour compter}

\section{Les manifestations pratiques de savoirs criminologiques dans les instances locales de sécurité 1}

\section{Élodie LEMAIRE, Laurence PROTEAU}

Élodie Lemaire est sociologue, post-doctorante à l'Institut des sciences sociales du politique de l'École normale supérieure de Cachan (ISP-ENS Cachan). Ses travaux portent sur les effets des réformes administratives sur l'institution policière, sur les procédures de construction des chiffres de la délinquance et sur les usages des technologies de surveillance dans le champ de la sécurité publique.

Laurence Protean est sociologue, maître de conférences à l'Université de Picardie Jules-Vernes (Amiens) et chercheur an Centre européen de sociologie et de science politique (CESSP-CSE/EHESS/Paris). Ses travaux portent sur l'espace des positions et des dispositions dans l'institution policière et sur les manifestations ordinaires des habitus et $d u$ sens pratique policiers.

Tes catégories de pensée et les méthodes de ce qui est présenté, par ses - représentants les plus emblématiques, Alain Bauer, Xavier Raufer et Christophe Soullez comme une "nouvelle criminologie 2 ", dont l'objectif serait de lutter contre les phénomènes criminels en les décelant précocement ${ }^{3}$, connaissent une large diffusion, au moins dans l'espace politico-médiatique. Il serait désormais possible de prédire les risques (par des savoirs chiffrés et des cartographies notamment) et de les réduire par le déploiement de dispositifs humains, de prévention situationnelle ${ }^{4}$ ou technologiques (comme la vidéosurveillance).

1. Nous remercions Laurent Bonelli et Blandine Leclercq pour leurs relectures attentives de ce texte et Tim Raynal pour sa contribution à l'enquête.

2. Mucchielli L., "Une "nouvelle criminologie" française. Pour quoi et pour qui ? ", Revue de sciences criminelles et de droit pénal comparé, 4, 2008, pp. 795-803.

3. Bauer A., Raufer X., Roucaute Y., "Une vocation nouvelle pour la criminologie ", Sécurité globale, automne 2008, p. 89.

4. La prévention situationnelle, importée des États-Unis, recouvre l'ensemble des politiques de sécurité qui prétendent réduire le passage à l'acte des délinquants potentiels, en modifiant l'environnement urbain et en déployant des dispositifs de surveillance. Sur ce sujet, voir notamment Bonnet F., "Contrôler des populations par l'espace ? ", Politix, 97-1, 2012, pp. 25-46. 
La pierre angulaire de cette vision du monde, qui se cherche une caution scientifique ou du moins rationnelle, est la production d'un «vrai » chiffre de la délinquance, dont les statistiques des services de police et de gendarmerie rendraient imparfaitement compte. Les chiffres n'ont pourtant en eux-mêmes aucun privilège d'objectivité, ils sont toujours le produit d'une construction ${ }^{5}$. Mais leur autorité (leur «magie sociale») est mobilisée par ces experts pour asseoir leur légitimité tant dans le marché lucratif de la sécurité locale que dans le champ politique, médiatique et même administratif. De là leurs efforts pour conquérir des positions dans des institutions en situation de produire des données alternatives, comme l'observatoire national de la délinquance et des réponses pénales (ONDRP) et dans celles capables d'en assurer la diffusion à des acteurs de terrain, comme le Conservatoire national des arts et métiers (CNAM), qui compte depuis 2009 une chaire de criminologie, confiée à A. Bauer ${ }^{6}$.

Les approches prônées par les tenants de cette criminologie s'invitent dans les instances locales de sécurité, comme les comités locaux de sécurité et de prévention de la délinquance ${ }^{7}$ et les cellules de veille ${ }^{8}$. En réponse à l'injonction à la co-production de la sécurité, ces dispositifs dits " partenariaux " ${ }^{9}$ ont été mis en place pour définir, suivre et évaluer les projets contractualisés, le plus souvent sous la forme d'un contrat local de sécurité. Leur développement s'est accompagné d'une intense activité d'État de promotion des outils valorisés par la "nouvelle criminologie ", à laquelle ses partisans ont activement participé. Le contrat local de sécurité était par exemple subordonné à un «diagnostic de sécurité » reposant sur l'évaluation locale de la délinquance, et le Guide pratique pour les contrats locaux de sécurité, réalisé en 1998 par l'Institut des hautes études de la sécurité intérieure (IHESI), incitait à construire des outils statistiques et à créer des observatoires locaux.

De fait, en 2011, nous avons observé ( $c f$. encadré 1) que les acteurs chargés de l'organisation et de l'animation des réunions avec les partenaires locaux que nous appellerons des « coordonnateurs de la sécurité » 10 - s'investissent

5. À la suite des travaux majeurs d'Alain Desrosières, les instruments de quantification ne peuvent plus être considérés comme neutres socialement et politiquement. $C f$. entre autres, Desrosières A., La politique des grands nombres. Histoire de la raison statistique, Paris, $\mathrm{La}$ Découverte, 1993 et Robert Ph., Aubusson de Carvalay B., Pottier M-L. et Tournier P., Les comptes du crime. Les délinquances en France et leurs mesures, Paris, L'Harmattan, 1994.

6. Voir l'introduction de Didier Bigo et Laurent Bonelli dans ce numéro.

7. Créés en 2002, les CLSPD sont présidés par les maires. Ils remplacent les conseils communaux de prévention de la délinquance (CCPD) mis en place en 1983.

8. Les cellules de veille sont des réunions organisées par les coordonnateurs de la sécurité auxquelles prennent part les "partenaires » des comités locaux de sécurité et de prévention de la délinquance. Elles peuvent être ouvertes à de multiples instances, se tenir en comité restreint, ou encore être organisées par secteur/quartier.

9. Voir Douillet A.-C., de Maillard J., «Le magistrat, le maire et la sécurité publique : action publique partenariale et dynamiques professionnelles ", Revue française de sociologie, 49, 2008, pp. 793-818 et Gautron V., "La coproduction locale de la sécurité en France : un partenariat interinstitutionnel déficient », Champ pénal/Penal Field, vol. VII, 2010 [en ligne]. 
dans la production de connaissances sur les lieux et les catégories de populations «à risque » en reproduisant localement les dispositifs promus à l'échelon national : observatoires locaux, données chiffrées, cartographies, élaboration de profils de populations. On pourrait penser que, conformément à la conception des partisans de la «nouvelle criminologie » comme science appliquée, les coordonnateurs de la sécurité répliquent les enseignements et les méthodes criminologiques pour évaluer et gérer le phénomène délinquant à l'échelon local. Cependant, ce postulat conclut trop vite au décalque parfait entre les recettes " prêtes-à-porter » conçues par ces experts et les pratiques mises en œuvre par les agents engagés dans les dispositifs locaux. Ceux-ci retraduisent dans une certaine mesure les catégories d'anticipation des risques de délinquance en fonction d'enjeux locaux et de la configuration dans laquelle ils exercent. En réalité, la réappropriation pratique de ces outils est motivée par d'autres raisons que leur prétendue efficacité. C'est ce que nous tâcherons d'éclairer ici, en prenant pour objet les logiques et les modalités selon lesquelles les coordonnateurs de la sécurité s'investissent dans la production de données chiffrées. Comment s'y prennent-ils pour construire leurs indicateurs ? Pourquoi cherchent-ils à se doter d'instruments de quantification ? Si des dispositifs sont bel et bien mis en place localement pour produire des chiffres, sont-ils réellement mobilisés dans l'action publique ?

\section{Encadré 1 : Quelques éléments sur l'enquête}

L'enquête sur l'espace de production des indicateurs de mesure de la délinquance a été menée dans quatre communes d'une même région de 2011 à 2013. Le matériel empirique est composé d'une quarantaine d'entretiens réalisés avec des partenaires des comités locaux de sécurité et de prévention de la délinquance et d'observations de leurs réunions. L'enquête a également cherché à comprendre la fabrique des indicateurs de mesure de la délinquance dans les principales instances productrices de données chiffrées (la police nationale, la police municipale, les établissements scolaires, les sociétés de transports en commun et les bailleurs sociaux).

Nous interrogerons les conditions sociales de diffusion et d'appropriation des outils quantitatifs dans les instances locales de la sécurité, puis nous analyserons les usages et les effets pratiques des chiffres, notamment sur les décisions et les dispositifs mis en œuvre à l'échelon local.

10. Nous reprenons ici la dénomination de Tanguy Le Goff : Le Goff T., «L'insécurité “saisie” par les maires. Un enjeu de politiques municipales ", Revue française de science politique, 553, 2005, pp. 415-444. 


\section{La pensée «bricoleuse »}

À la fin des années 1990, la mise en place de la nouvelle doctrine de police de proximité voulue par le gouvernement socialiste, inscrite dans l'exigence encore renouvelée de partenariat, redéfinit et élargit l'espace de surveillance. Cette doctrine impose les «bonnes pratiques » de production de la sécurité comme l'obligation de l'expertise (diagnostic), du partenariat (contrat, « culture » commune de la sécurité), de l'évaluation (indicateur, objectif assigné, résultat obtenu), de l'ancrage territorial de la politique de sécurité (« co-production » de la sécurité par des acteurs locaux, institutionnels, privés, élus). Dans les faits, la police de proximité a l'ambition d'étendre son champ de surveillance et d'intervention à quasiment tous les aspects de la vie publique et privée (conflit familial, de voisinage, d'école, de groupe, contrôle des véhicules, des lieux, des identités, des corps, recueil de renseignements...). Comme le dit sans détour le Centre d'études et de recherches sur les qualifications (Céreq) : «L'objectif visé est de rééquilibrer le dispositif d'ensemble par renforcement de son maillon faible, celui du contrôle social local, jugé insuffisamment développé face aux pressions de la demande et aux menaces de désagrégation de l'ordre social dominant 11 ». La place prépondérante prise par les questions d'insécurité dans les politiques publiques et la construction de la délinquance comme un problème social central de nos sociétés, depuis les années 1990, ont déjà été suffisamment analysées pour que l'on ait besoin de s'y attarder ${ }^{12}$. En revanche, il est intéressant d'insister sur les injonctions politiques au renforcement de la surveillance policière des territoires et des populations supposées «dangereuses » parce que c'est dans ce contexte que le partenariat prend son essor. Une circulaire validée lors du Conseil de sécurité du 9 mars 1998, relative à « la lutte contre les violences urbaines ", éclaire le consensus politique concernant le " grand danger » que représenterait un nombre croissant de quartiers populaires classés en zones « sensibles». La politique du ministre de l'Intérieur de l'époque, Jean-Pierre Chevènement, tient en plusieurs orientations que les préfets doivent inscrire dans le cadre général des Contrats locaux de sécurité (CLS) nouvellement créés ${ }^{13}$. L'une d'elles concerne le développement du partenariat dont un des objectifs est d'accroître le recueil et le croisement d'informations sur les populations pour anticiper les risques. Afin de constituer un « dossier technique » sur chaque quartier, il faut affiner le dispositif d'observation en associant police, gendar-

11. Le Céreq dépend du ministère de l'Éducation nationale, de l'enseignement supérieur et de la recherche et du ministère de l'Emploi, du travail et du dialogue social. Il fait un travail d'expertise publique par la réalisation d'études, l'évaluation de dispositifs et la formulation de propositions. La citation dans le texte est extraite de : Bref, 154, juin 1999.

12. Sainati G. et Bonelli L., La machine à punir. Pratiques et discours sécuritaires, Paris, L'Esprit frappeur, 2001 ; Mucchielli L., Violences et insécurité. Fantasmes et réalités dans le débat français, Paris, La Découverte, 2001 ; Bourgoin N., La révolution sécuritaire (1976-2012), Nîmes, Champ social éditions, 2013.

13. Sur l'émergence du champ de l'insécurité dans lequel sont pris les CLS et autres dispositifs publics, on se reportera à Bonelli L., La France a peur : Une histoire sociale de l'« insécurité ", Paris, La Découverte, 2008. 
merie et partenaires du quartier («municipalités, administrations, associations, responsables de services publics, de centres commerciaux,...») dans une cellule de veille qui doit actualiser cette masse de renseignements et « pressentir l'apparition de tensions ou de crises ».

En 2006, les contrats locaux de sécurité nouvelle génération (CLS NG) ont remplacé les contrats locaux de sécurité. Ils renforcent encore le ciblage des banlieues populaires et cherchent à favoriser le traitement des situations individuelles et familiales. Dans ce contexte, les coordonnateurs de la sécurité fondent le CLS NG sur un diagnostic local de sécurité (DLS) faisant état non seulement de l'évolution de la délinquance sur l'ensemble du territoire concerné mais également sur une approche statistique détaillée des quartiers. Les dispositifs d'expertise externe jouent un rôle important dans ces évaluations quantifiées ${ }^{14}$. Deux des communes enquêtées ont confié la réalisation du DLS à une expertise institutionnelle (l'Institut national des hautes études de la sécurité et de la justice - INHESJ - qui prend la suite en 2009 de l'IHESI et le Forum français sur la sécurité urbaine - FFSU 15) et une troisième à une expertise commerciale (VERES Consultants), même si la distinction est plus que floue. Généralement standardisé, ce diagnostic se compose d'un état des lieux/bilan des moyens et des actions de prévention mises en œuvre, des statistiques, collectées essentiellement auprès de la police nationale, des faits de délinquance sur le territoire et, pour finir, d'une synthèse des préconisations élaborées à partir des informations chiffrées. Le suivi des CLS NG est assuré par les comités locaux de sécurité et de prévention de la délinquance (CLSPD), qui succèdent aux conseils communaux de prévention de la délinquance (CCPD) en 2002 et par des cellules de veille regroupant les partenaires des conseils locaux en comité plus restreint. Au cours des cellules de veille, les coordonnateurs tentent de collecter les données chiffrées auprès des divers partenaires, mais en réalité, bien qu'officiellement un grand nombre d'instances ${ }^{16}$ y participe, seules les sociétés de transports, les bailleurs sociaux et les forces de l'ordre leur transmettent régulièrement les chiffres construits sur la base de leurs propres indicateurs. C'est aussi parce que cette activité de production statistique s'inscrit dans leur travail ordinaire qu'ils ont plus de facilité à être les bons élèves de ces dispositifs. À l'inverse, les travailleurs sociaux

14. Buffat J.-P, Le Goff T., "Quand les maires s'en remettent aux experts. Une analyse des liens entre les cabinets de conseils en sécurité et les maires ", Les cabiers de la sécurité intérieure, 50, 2002, pp. 169-196 ; Bonelli L., "Quand les consultants se saisissent de la sécurité urbaine ", Savoir/Agir, 9, 2009, pp. 17-28.

15. Ce forum de villes a été créé en 1992 comme émanation nationale du Forum européen sur la sécurité urbaine (FESU), lancé par Gilbert Bonnemaison. Regroupant des élus de tous bords, il dispense, contre rémunération, des conseils, des diagnostics, des formations et des diplômes ; réalise des études, des fiches pratiques et distribue des prix.

16. «Les forces de l'ordre (police municipale, police nationale, gendarmerie), les services de la justice (protection judiciaire de la jeunesse, parquet), les services des impôts, de l'inspection du travail, de l'éducation nationale, les organismes de transports, les bailleurs sociaux, l'observatoire local et national de la délinquance et leurs enquêtes de victimation et les régies de quartiers ", In Observation locale et politique de la ville : notes stratégiques et guide méthodologique, édité par le DIV, septembre 2008. [mis en ligne sur le site ville.gouv.fr] 
et les associations doivent engager un travail d'objectivation statistique spécifique pour nourrir ces réunions. Non seulement l'investissement n'est pas identique en raison de ce rapport différent à la logique de la mesure, mais s'ajoute à cette première différence le fait que les uns et les autres n'ont pas également intérêt à « l'échange ». Par exemple, les organismes de transport et les bailleurs sociaux tirent profit de l'engagement dans ces dispositifs partenariaux en créant des liens plus directs avec l'institution policière, suscitant ainsi une mobilisation plus forte des policiers en cas de besoin, telles que la prise en charge des plaintes des bailleurs sociaux et la sécurisation des bus.

«Maintenant on participe aux réunions avec la police nationale, municipale, le conseil général, la gendarmerie et l'Éducation nationale. De la part de la police, il y a une plus forte réactivité qu'avant. Les rassemblements sur la voie publique par exemple, c'était un problème pour nous. Le conducteur était pris de panique, pour lui, s'il y a rassemblement c'est forcément synonyme de jets de projectiles ou d'agressions. Donc on faisait des déviations l'après-midi. Toutes ces déviations qu'on faisait un peu avant n'importe comment, elles n'existent pratiquement plus. Quand on a un problème de sécurisation des bus, on appelle la police et ils envoient une équipe » (Abdel M., 41 ans, responsable prévention-ambiance-sécurité et lutte contre la fraude, société de transport en commun).

Le fait que certaines instances membres des comités locaux n'assistent pas aux réunions ou y délèguent des agents subalternes qui n'ont ni le savoir social, ni les informations permettant de compter dans ces groupes, témoigne d'un engagement en demi-teinte et d'une croyance très limitée dans l'intérêt $\mathrm{du}$ dispositif. Ce service minimum de certains «partenaires » est une des difficultés à laquelle sont confrontés les coordonnateurs. Dans les propos d'un correspondant de jour (médiateur), rapportés ci-dessous, on devine l'opposition entre les «bons » élèves - dans lesquels il se place sans conteste - et les "mauvais » élèves qui, en ne prenant pas le dispositif au sérieux, discréditent ceux qui s'investissent parce qu'ils en attendent quelque chose. Dans cette configuration, l'Éducation nationale est accusée de ne pas jouer le jeu, en refusant soit de s'investir soit de partager des informations.

«Tous ceux qui sont censés venir ne viennent pas aux cellules de veille. Les chefs d'établissements envoient souvent les CPE [conseillers principaux d'éducation] à leur place et quand ils viennent, ils ne donnent pas vraiment d'infos, ils viennent juste en chercher. Ils ne disent rien mais ils notent ce qu'on dit. Ça ne va que dans un sens. Ce qui fait qu'il n'y a pas vraiment d'échange, alors que c'est quand même le but » (Luc R., 36 ans, correspondant de jour - médiateur). 
Ces absences - qui ne se répartissent pas au hasard - et ces stratégies de rétention d'informations, ne facilitent pas la tâche des coordonnateurs pour collecter et centraliser les données chiffrées. L'ambition du croisement des données se heurte également à la non fiabilité des outils "donnés clés en main » qui, conjuguée à la faible compétence technique des coordonnateurs en matière de traitement statistique, repousse la possibilité de tracer le crime en dressant une cartographie qui le relierait à d'autres données sociales. C’est là, à n'en pas douter, pour les coordonnateurs un outil de pouvoir, de leur pouvoir sur le groupe, pouvoir de fabriquer un fichier «nouveau ", somme des fichiers singuliers des diverses instances représentées. Outil qui semble bien difficile à mobiliser et peu fiable.

« Pertinent à terme, ce logiciel [CORTO] devrait l'être, efficace non. On a de gros soucis avec ce logiciel mais il y a deux facteurs. Un, c'est que comme beaucoup de logiciels ils sont donnés clefs en main sauf que quand tu le mets en place en pratique c'est compliqué. Deux, on n'a pas été capable de le suivre très clairement. Mais sinon à terme, oui pour moi je le jugerai pertinent parce qu'il va nous permettre de réunir les données des bailleurs, de la gendarmerie, la police nationale, la police municipale, donc ça nous permettra justement de croiser les différentes données et pas uniquement, on est bien d'accord, sur les chiffres de la délinquance. Donc ça va me permettre, moi, effectivement d'avoir une traçabilité beaucoup plus importante et de me rendre compte quels sont les points chauds sur les différents quartiers. Oui à terme, mais aujourd'hui non!» (Jean G., 36 ans, agent contractuel, chargé de mission prévention-sécurité).

Deux observatoires de la délinquance et de la tranquillité publique étaient également censés permettre de collecter des chiffres. Là encore, les municipalités ont eu recours aux expertises publiques et privées. La création de l'un avait nécessité l'intervention d'un cabinet d'audit (Société Géo-Prévention) pour proposer des indicateurs que les partenaires des comités locaux avaient ensuite affinés collectivement lors d'une réunion, tandis que l'autre observatoire avait adopté la stratégie inverse : les coordonnateurs avaient construit des indicateurs lors d'une réunion en comité restreint (cf. encadré 2), avant de solliciter l'intervention du FFSU. Cependant, dans les deux cas les observatoires étaient en sommeil faute d'être alimentés régulièrement en données et de disposer de personnels qualifiés pour les traiter. 


\section{Encadré 2 : La fabrique d'un observatoire local}

La mise en place d'un observatoire local dans l'une des communes enquêtées est à l'initiative du supérieur hiérarchique d'Anne B., chef de projet sécurité-prévention en charge du CLS, Gaël H. (38 ans, fils d'un ingénieur militaire et d'une greffière, marié à une assistante sociale). Il est titulaire d'un baccalauréat ES et d'une maîtrise de droit public. Lieutenant aux RG depuis 2002, il prend la tête de la direction "sécurité, prévention et gestion des risques urbains » (DSPRU) de la municipalité en 2008. Dans le cadre de la création de cet observatoire, un étudiant inscrit en Master 2 de science politique a réalisé un stage et rédigé un rapport qui repose sur la technique du benchmarking ${ }^{17}$ concernant les villes françaises qui ont déjà mis en place un observatoire, sur un état des lieux des outils dont dispose la municipalité pour mesurer la délinquance (main-courante informatisée et tableaux statistiques de la police municipale et du centre de sécurité urbaine, compte-rendu des cellules de veille, fiches de signalement des médiateurs) et il a proposé des indicateurs (incendies - de véhicules, de poubelles, de biens publics ; jets de projectiles - sur les bus, les forces de l'ordre, autre ; dégradations - de mobiliers urbains, par tags et graffitis ; agressions sur la voie publique et rassemblements dans les halls d'immeuble). L'observation de la réunion dédiée à la sélection des indicateurs nécessiterait un long développement. On ne peut ici qu'esquisser des pistes qui mettent à l'épreuve certaines idées reçues.

La première suppose que les indicateurs sont co-produits, selon ce terme fort à la mode dans les dispositifs " partenariaux ». En réalité, dans ce cas, ceux qui discutent du choix des indicateurs sont exclusivement des agents de la municipalité. Les indicateurs sont donc élaborés en amont des réunions plénières et soumis à la validation ou non des "partenaires ». En effet, les indicateurs présentés au cours de cette réunion sont déjà utilisés par le directeur du service municipal en charge de la sécurité et de la prévention dans les cartographies de la délinquance dont il est l'auteur : "II s'agit de retenir tous ceux qui sont déjà dans la cartographie de la délinquance : tags et graffitis, jets de projectile, incendies de poubelles, incendies de véhicules, les dégradations sur l'espace public ». Ces indicateurs préétablis soumis à la validation des participants présents " aux origines », inscrits de par leurs fonctions dans un univers de rationalités et de croyances communes, seront par la suite imposés plus largement : "Bon, on va déjà commencer par ça et le présenter aux partenaires qui vont certainement vouloir qu'on quantifie autre chose. Là, il faut qu'on résiste aussi en disant que nous ne sommes pas en capacité pour l'instant d'intégrer d'autres choses ».

Une seconde idée reçue tombe à l'observation de cette réunion : la prétendue exigence méthodologique sur laquelle est censée reposer la construction des indicateurs. En réalité, les participants bricolent une liste (floue et mouvante) d'indicateurs sans réflexion critique. Les indicateurs des autres observatoires recensés dans le rapport du stagiaire ne sont même pas mentionnés. Le directeur de la DSPRU évoque les résultats de ce rapport uniquement pour souligner le temps que requièrent la création et le suivi d'un observatoire : " D'après le benchmarking qu'a fait déjà un peu le stagiaire, c'est $1 / 3$ de temps complet grosso modo pour faire vivre un observatoire de la délinquance, après la mise en route. ". Le choix des indicateurs est soumis à des considérations pratiques (qui va renseigner les indicateurs ?) et donc à l'impératif de simplicité pour permettre aux acteurs locaux de les renseigner plus aisément : " Moi, je pense qu'il faut commencer par des catégories générales et qu'une fois qu'on sera en période de croisière, on pourra l'enrichir d'autres rubriques. Après est-ce qu'on aura les moyens d'alimenter les différentes rubriques? Parce que si on détaille comme pour les jets de projectile, forces de l'ordre ou bus et si on veut être complet, véhicules de service public et ensuite privé etc., si tu veux développer comme ça, tu vas vite arriver à 15, 20 trucs à remplir. Donc il faudra qu'on se mette d'accord là-dessus et peut-être dans un premier temps qu'on renonce à un niveau de détail parce que ça peut faire peur aux partenaires qui doivent nous alimenter et qui vont se dire, donc là faut que je classe où ce truc-là, je sais pas! Donc voilà, il faut quelque chose de simple. II faut bien savoir qu'après il faut l'alimenter et que plus il y a de sous-rubriques, plus c'est compliqué à faire vivre. ». Par ailleurs, il n'a aucune certitude quant aux modalités de centralisation et de traitement des données recueillies : «(...) je présume que ce sera le chef de service avec sans doute une assistance administrative, quelqu'un de la CAF (Caisse d'allocations familiales), si on arrive à former quelqu'un et avoir quelqu'un pour le faire vivre ».

Outre les considérations pratiques, les faits de « délinquance » mis en chiffre sont ceux qui entrent dans ce que le directeur juge être du domaine de compétence de la municipalité et non de la police nationale : "Il y a un certain nombre d'informations pertinentes pour la PN mais qui ne se passent pas

17. Deux ouvrages permettent de mieux comprendre cette notion et les enjeux économiques, politiques et sociaux dans lesquels elle s'inscrit et notamment ceux qui commandent son importation de l'économie de marché vers les administrations publiques : Bruno I. et Didier E., Benchmarking. L'État sous pression statistique, Paris, Zones, 2013 ; Bezès P., Réinventer l'État. Les réformes de l'administration française (1962-2008), Paris, PUF, 2009. 
sur la voie publique et donc qui ne nous intéressent pas (...). Ensuite dégradations du mobilier urbain, dans lequel on va intégrer les caméras, les bancs, les vitres etc. Mobilier urbain pour moi, ce sont des choses qui appartiennent à la collectivité ou des édifices publics. Si on est d'accord là-dessus, on peut déjà valider ça ». À la question : "Mais tu as aussi des vols simples, tu as des vols sans violence. Vols de billets ou vols de portable, ça peut être sans violence. On n'intègre pas les vols simples ? ", formulée par la chef de la police municipale, le directeur répond: "Non, parce que c'est une soustraction, la personne ne s'en rend pas compte. Alors ça peut participer au sentiment d'insécurité, je suis d'accord avec toi, mais moi je ne les aurais pas retenus. D'abord, parce que je pense qu'on aura du mal à tout cartographier et est-ce que c'est le boulot de la ville, pour moi c'est plutôt le boulot de la PN (police nationale) ». Pour lui, la mise en chiffre de ces formes de délinquance permettra aux services de la municipalité d'agir là où la police nationale n'intervient pas et par voie de conséquence autorisera des opérations de communication politique des maires dans ce domaine : "Moi j'aurais bien aimé qu'on ait un truc spécifique sur les tags, parce que ça aurait pu conduire à une action rapide de l'unité anti-tags alors que si ce sont des dégradations, c'est pas le même service qui intervient. Pour moi, c'est intéressant de distinguer les tags, même si juridiquement ce sont des dégradations du mobilier urbain (...). Alors c'est pour ça qu'on s'interroge aussi s'il s'agit bien d'un observatoire de la délinquance, auquel cas, on ne doit faire apparaître que des actes de délinquance ou alors est-ce qu'on est sur un observatoire de la tranquillité qui nous permet d'intégrer des choses qui ne sont pas délictuelles. Par exemple, si on met en place un observatoire de la tranquillité publique, il n'y a pas forcément besoin de dépôt de plaintes et on peut surtout mettre des choses comme les rassemblements. Les rassemblements, c'est pas interdit donc on peut cartographier les rassemblements sans aucune difficulté. C'est intéressant aussi pour les médiateurs et pour la police municipale, ne serait-ce que pour aller faire de la médiation, de savoir où est-ce qu'on localise et à quels horaires on localise ces rassemblements. C'est évidemment pas un outil qui doit seulement servir à constater. Il faut apporter des mesures correctives en conséquence. L'idée c'est qu'on n'a pas des effectifs qui sont extensibles, donc en fonction des faits constatés sur le terrain, on adapte notre action à ce qu'on a constaté le mois précédent et pourquoi pas le jour précédent (...). La question est, est-ce que nous collectivité, on peut apporter des mesures correctives suite à ça. Je pense aux incendies de véhicules. On peut dire : on constate un grand nombre d'incendies de véhicules dans ce secteur, surveillons en particulier ce secteur-là. On peut avoir une action directe. Dégradation du mobilier urbain, on peut acheter du mobilier adapté. Les jets de projectile, on peut faire de la prévention situationnelle, renforcer la présence policière et la médiation de la police municipale et des médiateurs. Agressions sur la voie publique, pareil on peut renforcer la présence de la police municipale, des médiateurs et adapter la vidéo. Et rassemblement, là on voit tout de suite qu'en matière de médiateur et de police municipale, on aura une prise ».

L'absence de données ne serait pas le seul problème à résoudre pour alimenter ces observatoires, parce que même lorsque les diverses instances transmettent des chiffres qu'elles ont elles-mêmes produits, les situations hétérogènes qu'ils comptabilisent (incivilités, délinquance, tranquillité) et leurs modes de comptabilité font obstacle à la comparabilité des données. Pour le comprendre, nous proposons de restituer les conditions concrètes dans lesquelles s'effectue ce travail de production de données.

Les indicateurs recensés dans ces comités s'apparentent à un inventaire à la Prévert : «jets de crachat », " gêne volontaire par jet de lumière », «chahut », « altercations verbales », " mauvaises odeurs », " nuisances dues au comportement sur deux-roues motorisés », « absentéisme scolaire », « vols » " rassemblement d'individus qui provoque le sentiment d'insécurité », « occupation gênante », «souillures », «cambriolages ». Cette absence de taxinomie stable doit être rapportée non seulement à la forte segmentation du travail de production des données chiffrées, à l'éparpillement des intérêts des uns et des autres, mais également aux profils des agents impliqués dans la mesure, qui ne sont ni statisticiens professionnels, ni même formés à la statistique. 
Les bailleurs sociaux par exemple distribuent aux gardiens d'immeuble un carnet dans lequel figurent les principales infractions juridiques. Les gardiens renseignent les infractions qu'ils constatent dans des fiches informatisées. Ces fiches sont transmises à une unité en charge de la prévention et de la délinquance des bailleurs sociaux composée de deux secrétaires, de deux vidéo-opérateurs, d'un chargé de mission et d'un directeur. Ces «données » sont traitées par des secrétaires administratives qui reportent les informations dans un logiciel (logiciel business).

Dans les sociétés de transport en commun, ce sont les agents du poste de commandement, en liaison directe avec les bus, qui reportent les différents incidents constatés au cours de la journée dans un logiciel Excel dénommé « main courante » par les acteurs. Ce logiciel se compose de plusieurs rubriques à renseigner : le type d'incidents et les commentaires associés, le service du conducteur, son nom, le numéro du bus et de la ligne. Ces données sont centralisées et transmises au responsable prévention-ambiance-sécurité.

Bien que les agents du premier maillon de la chaîne (les gardiens d'immeuble, les agents du poste de commandement des bus, les secrétaires, etc.) disposent d'indicateurs préétablis, la plupart cochent la rubrique divers pour rapporter les faits constatés ou portés à leurs connaissances.

«Au cours de la journée, je prends des notes sur un papier pour ne pas oublier les incidents qui se produisent et dès que j'ai un peu de temps je rapporte les incidents dans la main courante. Je vais dans “type d'incidents” et je décris ce qu'il s'est passé. Bon, en définitive, on choisit souvent “divers”, parce qu'on n'est pas toujours sûr de bien choisir le type d'incidents. » (Josiane A., 51 ans, chargée du commandement des bus).

Ces « données » sont agrégées dans des indicateurs, issus soit d'une statistique standardisée descendante (fichiers de la police nationale et de la gendarmerie) soit inventés par les agents en charge de leur centralisation. Ceux qui mettent en chiffre la « délinquance » ne retiennent et ne comptabilisent donc pas les mêmes comportements comme dignes de figurer dans leur catalogue. En témoigne cette déclinaison sectorielle des écarts à la norme : les sociétés de transport répertorient le nombre d'incidents dans et aux abords des bus, les bailleurs sociaux recensent les troubles locatifs et les établissements scolaires quantifient l'absentéisme.

Les diverses pratiques mises en œuvres dans les instances locales de sécurité pour collecter des chiffres et construire des indicateurs relèvent plus sou- 
vent d'un bricolage armé de quelques notions floues que d'un véritable travail de construction de données quantitatives. La réflexion méthodologique est faible, voire inexistante et les chiffres ainsi produits ne sont pas le fruit de techniques statistiques testées et maîtrisées. De plus, ces dispositifs sont souvent peu renseignés faute de temps et de savoirs suffisants pour organiser le recueil et réaliser le traitement des données. Les projets mis en œuvre utilisent le chiffre plus comme façade et comme système de justification que comme élément probant. Ainsi, la construction d'outils à prétention criminologique dans les instances locales de la sécurité n'est pas le gage d'une connaissance scientifique de la délinquance à l'échelon local. L'absence de conventions techniques consolidées 18 et la faible maîtrise des logiciels empêchent la comparabilité des données chiffrées. En réalité, le déploiement des dispositifs de comptage est motivé par d'autres raisons que la «volonté de savoir».

\section{Conditions de l'adhésion aux techniques « criminologiques": positions floues et faible légitimité des coordonnateurs}

Pour rendre raison de la montée en puissance - apparente au moins - des données chiffrées sur « le risque » dans ces dispositifs, il faut s'intéresser aux intérêts des coordonnateurs à construire - ou à utiliser - ces outils. Habitués au cours de leur scolarité et au fil de leurs activités professionnelles à mobiliser les techniques quantitatives - et plus prosaïquement à agréger des données différentes dans des catégories mal délimitées-, comme une forme légitime de pensée du monde social, les coordonnateurs ont également tout intérêt à s'en saisir pour asseoir leur légitimité auprès de leurs employeurs, de leurs « partenaires » et pour s'assurer et se rassurer eux-mêmes. La crédibilité de leur poste repose, en grande partie, sur leur capacité à produire l'illusion que le regroupement d'institutions différentes dans un même dispositif apporte de la valeur ajoutée aux techniques de gouvernement local de la sécurité. Cette croyance, pour fonctionner, a besoin de s'incarner dans une œuvre commune qui serait plus que la somme des savoirs séparés des diverses instances. Or, comme nous l'avons déjà indiqué, l'accumulation d'indicateurs venant de diverses sources ne nourrit pas un savoir statistique consolidé. En revanche, elle fournit une caution apparemment scientifique à ces dispositifs et aux postes qui y sont attachés. Dans les quatre municipalités enquêtées, les coordonnateurs ${ }^{19}$ ont été exposés à ce type de techniques au cours de leur formation initiale et continue.

Anne B, 55 ans, a mené de front des études d'économie et de sociologie à l'université et a obtenu une maîtrise (aujourd'hui M1) dans

18. Crawford A., «Les politiques de sécurité locale et de prévention de la délinquance en Angleterre et au pays de galles : nouvelles stratégies et nouveaux développements ", Déviance et Société, 25, 2001, pp. 427-458.

19. Bailleau F. et Faget J. (ed.), Les " experts " municipaux de la sécurité. Origine, place et rôle dans la production locale de sécurité, CERVL/GRASS, Paris, IHESI, 2004. 
ces deux disciplines. Elle occupe depuis 2008 un poste d'attaché territorial et de chef de projet prévention-sécurité (fille d'ouvrier).

Madou O., 32 ans, a suivi des études de science politique. Après son DESS (aujourd'hui M2 pro), il a obtenu un poste d'attaché territorial et de chargé de mission prévention-sécurité qu'il occupe depuis 2011 (fils d'ouvrier).

Jean G., 36 ans, est titulaire d'une maîtrise en droit public. Depuis 2005, il est agent contractuel, chargé de mission prévention-sécurité (fils d'un ouvrier et d'une infirmière).

Jacques S., 33 ans est lui aussi titulaire d'une maîtrise de droit. Il est agent contractuel, chef de projet prévention-sécurité depuis 2012 (fils d'un dessinateur industriel).

Jean G. et Jacques S. ont suivi de surcroît une formation spécialisée, l'un dans la sécurité publique et l'autre, en criminologie.

En Master 2, Jean G. décide « de partir dans une filière plus spécialisée en droit privé notamment sur les questions de sécurité » et intègre en 2001 « le master de l’École Nationale Supérieure de Police à Lyon pour devenir commissaire ", avant d'être recruté comme chargé de mission prévention-sécurité.

Après l'obtention d'une maîtrise de droit pénal, Jacques S. tente « le concours d'École Nationale de la Magistrature pour devenir juge d'instruction »; il échoue. L'année suivante, il obtient un Diplôme Universitaire de sciences criminelles puis décide de poursuivre dans cette direction : « [...] sauf que voilà une fois lancé dans la recherche de formation en France, je ne trouvais pas forcément ce qui pouvait correspondre et en échangeant avec différents enseignants, ils m'ont indiqué qu'il valait mieux se tourner vers les pays étrangers donc soit le Canada, la Suisse ou la Belgique [...] je suis parti en Belgique pour faire un DESS en criminologie option sécurité urbaine ». En 2011, après son DESS, il débute une "formation en médiation au Conservatoire National des Arts et Métiers » qu'il abandonne rapidement suite à son recrutement comme chef de projet préventionsécurité.

Anne B. est la seule à avoir exercé d'autres activités avant de devenir chef de projet prévention-sécurité, mais dans un domaine très proche en lien avec la politique de la ville. Ses diplômes d'économie et de sociologie en poche, elle est d'abord recrutée comme chargée d'études par un bailleur social « pour réaliser des enquêtes sociolo- 
giques auprès des locataires ». En 1990, elle obtient un poste de chef de projet (Développement social des quartiers - DSQ) avant de passer le concours de la fonction publique territoriale en 1991. Elle exerce cette fonction pendant 15 ans, puis obtient en 2006 un poste de chargée de mission prévention-sécurité et intègre en 2008 la Direction "sécurité, prévention et gestion des risques urbains », frâichement créée, en tant que chef de projet prévention-sécurité.

Ces coordonnateurs ont été confrontés aux outils quantitatifs au cours de leurs cursus scolaires et/ou de leurs expériences professionnelles antérieures. Censés élaborer et évaluer les stratégies de sécurité et de prévention, ils suivent pour cela des formations assurées par le FFSU et le Centre national de la fonction publique territoriale (CNFPT), qui non seulement valorisent cette appréhension comptable du monde social mais définissent également les «bonnes pratiques » en matière de production statistique. Le FFSU dispense par exemple une formation intitulée «Diagnostic et stratégie de sécurité et de prévention » censée aider les coordonnateurs à se doter « d'outils de diagnostic et d'évaluation » pour "définir une stratégie de sécurité et de prévention » 20 .

Ces coordonnateurs ont également tout intérêt à se saisir de ces outils pour consolider la légitimité de leur poste. En effet, ce métier est relativement nouveau et largement dominé par rapport aux autres professions impliquées dans l'espace local de la sécurité ${ }^{21}$. On ne peut même pas dire d'eux, d'ailleurs, qu'ils forment une profession et c'est bien là un des éléments qui explique leur attachement à la mesure qui produit un « effet de science » et aux références criminologiques qui semblent encadrer des pratiques disparates et encore peu formalisées. Privés des moyens d'investir dans les savoir-faire professionnels et politiques des acteurs qui les entourent, comme les élus locaux ou la police nationale, dont l'autorité repose sur la légitimité de l'élection dans le premier cas et sur le savoir-faire sécuritaire institutionnalisé de longue date dans le second, les agents chargés de coordonner les dispositifs de sécurité s'approprient des savoirs et des outils à prétention criminologiques pour garantir leur expertise et tenter de s'imposer dans cet espace. En témoigne la manière dont Jacques S. présente la valeur ajoutée de son diplôme en criminologie qui, pour lui, fait la différence.

« J'ai des diplômes dans la criminologie et la sécurité urbaine. Ce sont des formations importantes car je pense que la préventionsécurité urbaine est un domaine assez spécifique. C'est-à-dire que

20. Voir le site de la FFSU : ffsu.org

21. Sur le malaise des coordonnateurs voir : "Contrats Locaux de Sécurité et Conseils Locaux de Sécurité et de Prévention de la Délinquance : bilans et perspectives », Enquête réalisée par la FMVM du 13 au 28 juin 2005 ; CNV, Synthèse et analyse de la consultation des coordonnateurs de CLSPD/CISPD, 2011. 
les phénomènes de délinquance sont complexes et qu'une seule formation en politique de la ville ne peut pas t'aider à bien appréhender tout ce qui va permettre de les expliquer. Si tu n'as pas de connaissances de ces phénomènes, des comportements délinquants, des lieux de délinquance, tu ne peux pas accomplir tout ce travail qu'on mène avec les partenaires » (Jacques S., 33 ans, agent contractuel, chef de projet prévention-sécurité).

On voit aussi dans ces propos que la mobilisation d'une forme appliquée des techniques d'experts criminologiques se comprend dans un espace de concurrence entre agents susceptibles d'occuper ces positions. En effet, les formations dans le domaine de la politique de la ville ne seraient plus suffisantes, selon lui. L'attachement à cette forme de technologie criminologique peut donc être analysé comme un double coup de force : se débarrasser d'une partie des concurrents aux postes de coordonnateurs et briller à cette position en s'imposant comme savant aux yeux des autres professionnels. Le choix du type d'information abordée et d'indicateur retenu lors des cellules de veille en atteste. Lors des réunions animées par les coordonnateurs, ces derniers orientent les discussions sur les « faits de délinquance supposés ou craints » et valorisent l'apport des données chiffrées produites sur les formes de déviance (incivilités, absentéisme, nuisances, désordres) pour prétendre à une vision globale de l'insécurité locale. Pour prendre la mesure de ce tour de force, il faut restituer les oppositions structurantes de cet espace. Les professionnels qui participent aux cellules de veille ne partagent pas la même appréhension de l'insécurité. Ceux qui dominent le champ de la sécurité publique (police et gendarmerie) privilégient les catégories d'infractions officielles tandis que « les nouveaux entrants » (associations, travailleurs sociaux) tentent de mettre en avant des catégorisations concurrentes comme les incivilités, par exemple. Cette opposition, qui structure l'espace, se retrouve dans les pratiques et les visions du monde des divers «partenaires », ce qui montre bien les limites du discours qui vante le partenariat comme mode de gouvernement coopératif. Le tableau 1, ci-dessous, synthétise l'opposition entre un pôle sécuritaire (forces de l'ordre) et un pôle préventif, mais aussi quasi prédictif (travailleurs sociaux).

Tableau 1 : Structure de l'espace de pensée et de prise en charge de la " délinquance »

\begin{tabular}{|c|c|c|}
\hline & Normes juridiques & Normes morales \\
\hline Faits constatés & Dégradations & Mauvaises odeurs \\
\hline Faits supposés ou craints & $\begin{array}{l}\text { Occupation abusive } \\
\text { de halls d'immeubles }\end{array}$ & Incivilités \\
\hline
\end{tabular}


La mise en évidence de ce système de classement permet de mieux cerner la configuration de l'espace de production des comités locaux en comprenant ce qui autorise les affinités et explique les alliances.

C'est par exemple auprès de la police nationale que les bailleurs sociaux siègent dans les cellules de veille et cherchent à obtenir des renseignements. Ces deux groupes s'accordent sur l'intérêt d'aborder les faits de délinquance constatés, comme les dégradations, et d'échanger des informations sur les auteurs potentiels. En revanche, c'est avec les membres des associations ou les assistances sociales scolaires que les travailleurs sociaux en commissariat échangent des informations.

Cela permet également d'expliquer ce qui concentre les rivalités et durcit les conflits de territoire. En témoigne la manière dont Hervé E., 48 ans, brigadier-major de la police nationale, évoquait lors d'une cellule de veille les initiatives proposées par les associations et les centres sociaux : «La dernière fois l'association Voyage vient nous parler d'un programme de vacances d'été. C'est la meilleure ! Je crois que là il faudrait réorienter un peu la cellule sur les questions de sécurité. Ça tourne mal ». En conséquence, l'espace de pensée et de prise en charge de la délinquance se structure autour de ces deux pôles, de ces catégorisations concurrentielles, qui recoupent une division sociale du travail du contrôle social dans laquelle chacun tente de préserver son territoire, d'affirmer son expertise et construit sa population cible (les délinquants/les déviants).

S'il est trivial d'identifier ce schéma général d'appréhension, ce qui l'est moins en revanche, c'est d'observer la montée en puissance d'un pôle d'épidémiologie de la sécurité, porté en grande partie par les coordonnateurs qui agitent les « bonnes pratiques » en matière d'anticipation et de gestion des risques de délinquance pour asseoir leur position. Dans cette configuration, si les chiffres de la police ne sont pas contestés, ils ne suscitent pourtant pas l'intérêt pratique des coordonnateurs : « Les chiffres de la PN (police nationale), bon, ça nous sert pas à grand-chose finalement, ça donne juste une idée de ce qui se passe comme faits marquants. (...) Mais bon, c'est un peu comme un passage obligé dans les cellules de veille » (Julien N., 38 ans, correspondant de jourmédiateur). En revanche, les données relatives aux désordres locaux, par exemple, constituent un corps de connaissances prisé, comme l'affirme Anne B., chef de projet prévention-sécurité :

«L'insécurité ce ne sont pas que les actes de délinquance qui sont relevés par les forces de polices, les délits... mais il y a aussi le sentiment d'insécurité, c'est beaucoup plus large. Il y a des gens qui ont un sentiment d'insécurité alors qu'il n'y a pas forcément que des 
faits qui sont de l'ordre du délit pénal. [...] C'est ce que je cherche à chiffrer, mais ce n'est pas évident ». (Anne B., 55 ans, attachée territoriale, chef de projet prévention- sécurité).

En témoignent les catégories comme les incivilités et l'absentéisme, retenues par les coordonnateurs dans les fiches qu'ils élaborent. Ces indicateurs de déviance sont construits moins selon des critères strictement policiers qu'en référence à des normes morales communes opérant le tri entre «bonne » et «mauvaise » jeunesse et qui représenterait un danger. Les coordonnateurs qui animent les réunions font la part belle à la prévention situationnelle et aux informations relatives aux « risques » de délinquance que détiennent les autres partenaires (les jeunes qui «vont mal tourner », les lieux qui « risquent » d'être dégradés, "les conditions pour voir apparaître des comportements délinquants »), qu'il faut traiter et réprimer avant qu'ils ne conduisent à des faits plus graves 22. L'intérêt porté aux petits délits et aux « risques de délinquance » ${ }^{23}$, au détriment de la délinquance complexe et de la « délinquance avérée ", suggère l'intériorisation pratique du paradigme de la vitre cassée 24 dans l'espace des comités locaux, comme le confirme le représentant d'un bailleur social :

"Quand on a démarré en 1998, pour les chefs d'établissements et les associations, c'était Peace and Love. C'étaient des petits qui faisaient des petites bêtises. Aujourd'hui ils ne sont plus du tout dans ce registre. J'ai vu des gens de l'Éducation nationale évoluer complètement. Le registre de la répression ne les effraie plus du tout. Le mot répression ne les effraie plus. Ils sont les premiers à appeler la police nationale quand ça tourne mal » (Henri L., 48 ans, représentant d'un bailleur social).

Les coordonnateurs tiennent le rôle principal dans la construction de cette « représentation commune », selon Jacques S. :

«Notre rôle, c'est de créer ce qu'on appelle une représentation commune. Si on veut pouvoir travailler sur un sujet commun, sachant qu'on est tous d'horizons différents, qu'on a tous des missions différentes, qu'on a tous des contraintes différentes et des objectifs différents, il faut à un moment donné qu'on ait la même image, la même représentation de ce qu'on voit et de ce qu'on fait » (Jacques S., 33 ans, agent contractuel, chef de projet prévention-sécurité).

22. Bonelli L., Sainati G., La machine à punir..., op. cit., p. 31.

23. Bailleau F., "Prévention de la délinquance ou gestion du risque ? Un changement de paradigme ", Les Cabiers Dynamiques, 51, 2011, pp. 6-15.

24. Wilson J. Q. et Kelling G. L., «Les vitres cassées », in Brodeur J.-P., Monjardet D. (ed.), Cabiers de la Sécurité Intérieure, hors-série, 2003, pp. 233-255. 
Le coordonnateur ne serait pas seulement un animateur, mais chercherait à homogénéiser, avec plus ou moins de succès, l'espace des points de vue; du moins proposerait-il une vision acceptable par les différents partenaires. Les catégories criminologiques d'anticipation des risques de délinquance sont ainsi pour lui un moyen de dépasser les expertises singulières en proposant une vision, au-delà du phénomène délinquant, de l'insécurité locale, prétendument plus complète.

Ainsi les chiffres sur les désordres locaux, bricolés à partir des données transmises par les diverses institutions, permettent aux coordonnateurs d'avoir une raison d'exister dans cet espace, en complétant voire en opposant à la connaissance de la délinquance, monopolisée par les institutions régaliennes, celle plus large des phénomènes susceptibles d'entretenir le sentiment d'insécurité, mais également de satisfaire les attentes des maires, qui utilisent les chiffres dans leurs opérations de communication. Le succès des chiffres doit donc être rapporté aux stratégies de légitimation des coordonnateurs et aux opérations de communication politique que ces outils autorisent. On voit là comment se fabrique un coup symbolique dans un espace dit "partenarial » : ceux qui sont en réalité dépourvus de la garantie qu'offre une profession reconnue vont puiser dans des «savoirs " à la marge de la science, mais qui ont le vent en poupe politiquement, pour affirmer leur expertise et ainsi légitimer leurs postes et leurs manières de voir.

\section{L'individualisation du traitement local de la délinquance et de la déviance}

Paradoxalement, l'investissement des coordonnateurs dans la production de données chiffrées n'implique pas qu'il en soit fait usage systématiquement, loin s'en faut, dans la gestion locale de la sécurité. En effet, les actions engagées ne reposent pas sur des chiffres, mais sur des informations qualitatives et nominatives : lieux, horaires et parfois nom et caractéristiques de l'auteur de l'acte. En conséquence, si les techniques criminologiques qui produisent des indicateurs quantitatifs sont mises en œuvre comme catégories de pensée, elles ne sont pas pour autant utilisées comme catégorie d'action. Alors à quoi servent-elles ? Elles permettent indirectement de légitimer le resserrement du contrôle social à l'échelle locale par la mise en commun d'informations nominatives sur des individus jugés à « risque ». Ces informations qualitatives intéressent particulièrement les coordonnateurs. On le voit bien dans la manière dont Anne B., chef de projet, choisit les «partenaires » des comités locaux : «Je pense que pour les prochaines réunions, il faudrait associer la PJJ. Ce sont eux qui ont la liste des sortants de prison et comme la police ne les donne plus... Il faudrait convier quelqu'un qui suit de près les gamins pour qu'il puisse donner les bonnes informations, qu'il échange des informations nominatives. C'est bien de savoir qui va sortir, si c'est quelqu'un qui a fait des feux de poubelles par exemple. Ça nous permettra d'anticiper » (Anne B., 55 ans, 
attachée territoriale, chef de projet prévention-sécurité). En témoigne également le souci d'obtenir des données qualitatives, que ce soit dans les fiches distribuées par les chargés de missions ou dans les logiciels (géo-prévention) des observatoires locaux. En effet, les partenaires qui doivent renseigner les fiches élaborées par les coordonnateurs sont censés indiquer le nombre de faits, par exemple « dix feux de poubelle », mais ils sont également incités à ajouter la date, le lieu, et donner des éléments de contexte dans la colonne « synthèse des faits » prévue à cet effet. Les observatoires locaux sont également dotés de logiciels composés de rubriques permettent de recenser non seulement le nombre de faits commis, mais également le lieu précis (numéro de voirie), l'heure et les informations relatives à l'auteur de l'acte.

Lors des réunions, le chiffre est donc relégué à l'arrière-plan au profit des informations qualitatives - et le plus souvent nominatives - qui lui sont associées : «Le but dans les cellules de veille c'est d'arriver à échanger, sans tabous, sur des situations concrètes par exemple... donc ça nécessite d'avoir créé une relation de confiance [...], on cite des noms, donc il y a vraiment de la confiance entre nous » (Jean G., 36 ans, agent contractuel, chargé de mission prévention-sécurité). Cette stratégie révèle l'une des vertus supposée de la statistique : celle de «parler un langage commun » et de fournir une base consensuelle de discussion à partir de laquelle les «vrais problèmes » peuvent être abordés «sans tabous ». De fait, après l'exposition des chiffres des forces de l'ordre, les coordonnateurs procèdent, selon leurs propres termes, au rappel des faits marquants et des points sensibles identifiés le mois précédent. Pour cela, ils utilisent un document distribué à tous qui répertorie les lieux qui posent problème et les informations délivrées par les «partenaires » concernés.

Quartier Nord galerie commerciale. Éléments du bailleur social : pas de remontée d'information concernant la galerie marchande, par contre des regroupements conséquents devant le magasin de chaussure constatés. Police municipale : actions menées par la PN et les CRS qui ont dû faire usage de gaz lacrymogène le 16 mars lors de l'interpellation d'un individu porteur de produits stupéfiants. Présence journalière pendant 15 jours.

Quartier Est - acte de vandalisme, feu de poubelles, troubles de voisinage - rue Bil/5, rue Cagne. Police municipale ouest : il semblerait que les agissements du jeune $\mathrm{X}^{25}$ ont repris. Concernant ces faits, aucun appel, ni acte de dégradation enregistré ou constaté. Des passages sont assurés - RAS. La présence de ce jeune amène une inquiétude pour le voisinage qui craint de revivre les problèmes [actes de

25. Si les informations sont nominatives dans les réunions, aucun nom de famille n'apparaît cependant dans les comptes rendus des cellules de veille. 
vandalisme] qui ont eu lieu de juillet à décembre 2012. La PM reste vigilante et à l'écoute (Extrait du document répertoriant les faits marquants et les points sensibles, Cellule de Veille Ouest, 16 mai 2013).

Ils orientent ensuite les discussions vers les nouveaux faits que les « partenaires » souhaitent aborder et qu'ils exposent tour à tour. Les coordonnateurs procèdent à une description précise, et le plus souvent personnalisée, des « situations de délinquance ». Ils prennent ce qu'ils appellent la « température du terrain » et identifient des lieux sensibles ou les points chauds, mais également des familles «à problème »: «On a rencontré un problème de bagarre avec [tel jeune à tel endroit] »; «On a rencontré un problème de dégradation avec [telle famille dans tel immeuble] ». Cette logique du traitement d'espèces, de cas singuliers, contourne la mise en équivalence statistique et la «moyennisation » des écarts à la norme et plus exactement aux normes. Sont mises sur le même plan des infractions comme les feux de poubelles, les cambriolages, les violences intrafamiliales et des incivilités ou des nuisances.

Les décisions relatives par exemple au choix des territoires éligibles pour constituer le ressort géographique du CLS reposent moins sur les statistiques de la délinquance recensées dans le diagnostic local de sécurité que sur les informations qualitatives transmises par les " partenaires » locaux lors des cellules de veille. En témoigne cet extrait d'un contrat local de sécurité nouvelle génération daté de 2007 : «Six secteurs sont particulièrement touchés par les problématiques de délinquance et peuvent être à ce titre éligible au titre du CLS : le quartier [Saint Luc] qui, en terme statistique, n'est pas le principal secteur touché mais nécessite une attention particulière du fait des problématiques remontées lors des réunions de la cellule de veille ». C’est également sur la base de ces informations que des actions sont décidées. Le plus souvent, elles se réduisent à la mise en place de patrouilles de police sur ces points, à la saisie des médiateurs pour obtenir davantage d'informations, à l'intervention de spécialistes de l'aménagement du territoire ou d'architectes et, enfin, à l'engagement de certains partenaires, la plupart du temps la police nationale, à se renseigner sur telle et telle personne qui pose problème. Pour se sentir à sa place dans ces réunions et lors des échanges avec les partenaires, il faut disposer d'informations qui rencontrent l'intérêt des autres participants et ainsi pouvoir revendiquer d'en obtenir d'autres en retour. C'est donc plus la détention d'informations que la production de chiffres qui fonctionne comme un capital 26 et une ressource distinctive : observer les relations entre les partenaires, c'est observer des rapports inégaux de production et de captation de renseignements. 
Les effets pratiques de ces dispositifs partenariaux méritent attention car cette extension du contrôle social affecte la vie quotidienne de certains groupes sociaux. Les dispositifs partenariaux concourent moins à une vision totale de la population qu'à une vision hautement sélective ${ }^{27}$. Autrement dit, les groupes sociaux ne font pas l'objet d'un même contrôle et cette inégale invention de catégories de populations à risque enregistre et renforce encore les inégalités sociales préexistantes. Cet effet peut-être très concret, par exemple lorsque cette désignation réduit les chances d'accéder à certaines ressources (un logement, une école...). Roger R., responsable prévention-sécurité d'un bailleur social, ne laisse planer aucun doute sur la performativité de ces classements : «On peut discuter de la manière dont on peut faciliter le relogement de certains ou à l'inverse, de prévenir d'autres bailleurs en leur expliquant qu'on expulse telle famille et qu'il ne faut pas la prendre parce que c'est la garantie que quinze jours après son immeuble sera mis à feu et à sang "; «Le maire peut dire [aux familles qu'il a convoquées sur la base des informations nominatives échangées lors des cellules de veille],"dites-moi, là mon CCAS [centre communal d'action sociale], il vous aide tous les mois à payer votre chauffage et votre électricité, etc. Vous foutez le bordel dans votre immeuble et les enfants posent problème à l'école. Bon, écoutez, je ne vais pas pouvoir continuer une aide qui manifestement ne sert à rien” 》 (Roger R., 37 ans, responsable prévention-sécurité, bailleur social).

Le fait que les établissements scolaires, les bailleurs sociaux, entre autres, partagent et détiennent des informations, autrefois monopolisées par les forces de l'ordre, participe à nourrir la dimension cumulative des « handicaps » sociaux. Ainsi, ces dispositifs partenariaux recomposent les formes d'encadrement des classes populaires 28 et reproduisent des inégalités sociales, en habillant leurs pratiques des atours scientifiques des catégories et des méthodes d'une criminologie que nous appelons épidémiologique.

Notre propos peut être résumé simplement dans un mouvement en trois temps. Tout d'abord, l'espace de production de discours et de pratiques - qui est aussi un espace de positions - dominant au moins politico-médiatiquement aujourd'hui se rattache à un courant (faible en nombre et en contenu) qui tend à s'approprier le label « criminologie » pour faire reconnaitre son expertise dans le champ des technologies de pouvoir d'État. Si ce courant s'impose malgré sa faiblesse scientifique et numérique, c'est qu'il s'inscrit dans un espace plus vaste de technologies du pouvoir qui toutes, quel que soit leur domaine d'application, mobilisent les mêmes schèmes de pensée du monde social et les mêmes outils. Structurellement, ces schèmes de pensée visent à « contenir

27. Lyon D., Surveillance Society: Monitoring Everyday Life, Buckingham, Open University Press, 2001.

28. Actes de la recherche en sciences sociales, 136-137, 2001. 
l'avenir », voire à le prédire et, dans tous les cas, à le façonner. Comment se réalise cette ambition ? Par la construction d'indicateurs qui mesurent le risque d'être délinquant, d'être mère " précocement ", d'être maltraitant ou maltraité, d'être alcoolique ou diabétique, d'être en échec scolaire, d'être au chômage, d'être suicidaire, etc. Les technologies politiques de gestion du crime (ou des indisciplines) ne sont pas différentes d'autres technologies qui concernent d'autres domaines; bien au contraire, elles existent et prennent forme et force dans un mode de gouvernement ${ }^{29}$ et dans la fin d'un modèle social 30 .

Dans un second temps, ces « grands points de vue », en raison de leur médiatisation et de leur capacité à diffuser leurs recettes dans des instances publiques (ONDRP, INHESJ, CNAM, etc.) ou para publiques (FFSU), sont en position dominante pour proposer leurs mallettes pédagogiques clés en main ou pour former les futurs agents de terrain qui organisent la gestion de la sécurité. Mais dans ce cheminement, il y a des ajustements obligés entre la doctrine du « risque » et les usages qui en sont faits ; les pratiques sont moins conformes et homogènes que le laissent penser les plaquettes publicitaires des CLS. Si des dispositifs de comptage sont bel et bien mis en place localement, cela ne veut pas dire qu'ils influencent les actions mises en œuvre.

Néanmoins, même s'il y a du jeu et si les discours et méthodes venant du haut semblent plus fragiles une fois mis en pratique, il n'en reste pas moins qu'ils pénètrent ces instances partenariales, justement parce que les coordonnateurs - composés de professionnels divers -sont le plus souvent, en manque de légitimité. Ils ne sont spécialistes de rien, n’ont pas de métier propre à faire valoir. C'est certainement ce qui les dispose - avec la formation pour certains - à se saisir des rares outils disponibles, aussi indigents soient-ils. La nouveauté de leur position, le flou de leur poste, l'hétérogénéité de leurs formations et de leurs trajectoires, les poussent à rechercher un cadre unificateur sur lequel s'appuyer pour se structurer en tant que "profession » reconnue et également pour s'imposer dans les assemblées qu'ils doivent coordonner. S'ils ne veulent pas être de simples secrétaires ou des organisateurs de séminaires, ils n'ont d'autres choix que d'investir dans un domaine qui semble leur conférer une forme de capital propre. C'est parce qu'ils sont dominés qu'ils ne résistent pas ou peu à l'imposition d'une boîte à outil et d'un lexique commun ; qu'ils assimilent ou mobilisent sans distance critique les préceptes généraux qui hantent les « Guides pratiques » et autres bibles de l'intervention en situation partenariale. Et les élèves les plus appliqués de la doctrine sont généralement ceux qui ne peuvent se prévaloir d'une autorité locale propre, généralement constituée dans des positions professionnelles antérieures.

29. Bezès P., Réinventer l'État..., op. cit.

30. Castel R., Les métamorphoses de la question sociale. Une chronique du salariat, Paris, Fayard, 1995. 
En revanche, et c'est le troisième temps de notre raisonnement, quoique les coordonnateurs retiennent des méthodes, des outils, de l'idéologie sousjacente, quel que soit leur degré d'application (dans les deux sens du terme), le système de classement des populations et de perception du monde social qui sous-tend cette technologie du pouvoir, lui, est pérenne parce qu'il est désormais répandu bien au-delà des dispositifs qui nous retiennent ici. Ce système industrialise la construction de catégories « à risque » et légitime l'anticipation comme mode de lutte contre tous les «maux ». Au niveau local, ces catégories prennent corps dans des individus singuliers, le danger porte un nom de groupe, mais aussi un nom de famille ; l'anticipation, c'est l'information, laquelle localement, quand l'État est au guichet, désigne autant un habitant qu'un habitat.

Ce qui pêche dans l'imposition de cette nouvelle catégorie de l'action publique, ce n'est pas une mauvaise articulation supposée entre une doctrine «criminologique » qui serait efficace et juste et son application floutée par des exécutants simplement mal formés. C'est bien les présupposés constitutifs de la doctrine qui sont à remettre en cause complètement. La vision du monde cachée derrière l'apparente et rassurante neutralité du chiffre a une force de persuasion et se diffuse largement malgré la nullité de ses registres de preuve, parce qu'elle se coule dans une forme de pensée en termes de risque qui, comme l'a montré Castel, fait l'économie de la dangerosité avérée pour proposer une gestion/contrôle des populations qui présenteraient des " risques » de dangerosité ${ }^{31}$. La différence est décisive parce qu'elle change non seulement l'appréhension et la définition des populations, mais aussi les modes de gouvernement de ces catégories prétendument à risque. Des indicateurs de risques sont construits, des experts cultivent la catégorie qui les fait naître, des « destins » peuvent même être régulés avant d'advenir. La structure qui soustend les catégories de l'entendement de cette «nouvelle » criminologie comme technologie préventive de gouvernement du risque la dépasse largement et c'est bien pour cela qu'elle se diffuse aussi facilement : elle s'inscrit dans un espace de pensée d’État légitimant des formes de gouvernement qui organisent la répression avant le passage à l'acte par l'élaboration d'indicateurs de risque. Ce qui, bien évidemment, suppose la surveillance et le dépistage dans le domaine de la délinquance, mais également dans celui de la santé, de la protection sociale, de l'éducation, etc. Ainsi s'impose progressivement, depuis le milieu des années 1970 et plus encore depuis une vingtaine d'années, une épidémiologie gestionnaire comme catégorie de l'action publique 32 et plus largement comme catégorie de pensée du monde social.

31. Castel R., «De la dangerosité au risque », Actes de la recherche en sciences sociales, 47-48, juin 1983, p. 122.

32. Mucchielli L., "Vers une criminologie d'État en France ? Institutions, acteurs et doctrines d'une nouvelle science policière », Politix, 89-1, 2010, pp. 195-214. 